\title{
New High-Directivity Coupler Design With Coupled Spurlines
}

\author{
Sheng-Fuh Chang, Jia-Liang Chen, Yng-Huey Jeng, and Chain-Tin Wu
}

\begin{abstract}
A new high-directivity coupler using coupled spurlines is proposed. The effective capacitances of coupled spurlines generate an isolation zero at any desired frequency due to their equalization effect on the odd-mode and even-mode phase velocities. The measurement results demonstrate an improved performance over the conventional parallel-line coupler in terms of the isolation and the coupling flatness.
\end{abstract}

Index Terms-Microstrip directional coupler, parallel-line coupler, spurline.

\section{INTRODUCTION}

$\mathbf{M}$ ICROSTRIP directional couplers with conventional parallel-coupled lines are widely used in baluns, filters, and various microwave integrated circuits. However, their directivity deteriorates with frequency, which is resulted from the difference of the even-mode and odd-mode phase velocities. Various compensation techniques have been reported, which include adding a different dielectric overlay on the top of coupled lines [1] and wiggling the edges of coupled lines [2]. The compensation is also effective by adding reactive lumped elements to the coupled lines. Examples are the insertion of capacitors at the ends or the center of coupled lines [3] and the insertion of inductors between the collinear ports of coupled lines [4]. More than 30-dB improvement of directivity, compared with the uncompensated coupler, was reported. These techniques have drawbacks of either departing away from the planar structure, due to the addition of lump elements, or requiring special fabrication procedures for another dielectric overlay or wiggling the conductor edges.

In this letter, another new type of high-directivity coupler is proposed, which is composed of two coupled spulines inter-connected with a section of coupled microstrips. The enhancement of the directivity is resulted from the generation of an isolation zero by the coupled spurlines. A spurline coupler was designed and measured to verify the proposed technique.

\section{Generation of Isolation Zero by Coupled Spurlines}

The schematic of the proposed spurline coupler is shown in Fig. 1(a). This four-port network is reduced to the respective two-port even-mode and odd-mode networks, as shown in Fig. 1(b), by exciting the even-mode and the odd-mode voltage at two collinear ports. These reduced two-port networks include

Manuscript received July 1, 2003; revised September 17, 2003. The review of this letter was arranged by Associate Editor Rüdiger Vahldieck.

The authors are with the Center for Telecommunication Research, Department of Electrical Engineering, National Chung Cheng University, Chiayi 621 ,

Taiwan, R.O.C. (e-mail: ieesfc@ccu.edu.tw).

Digital Object Identifier 10.1109/LMWC.2003.822565

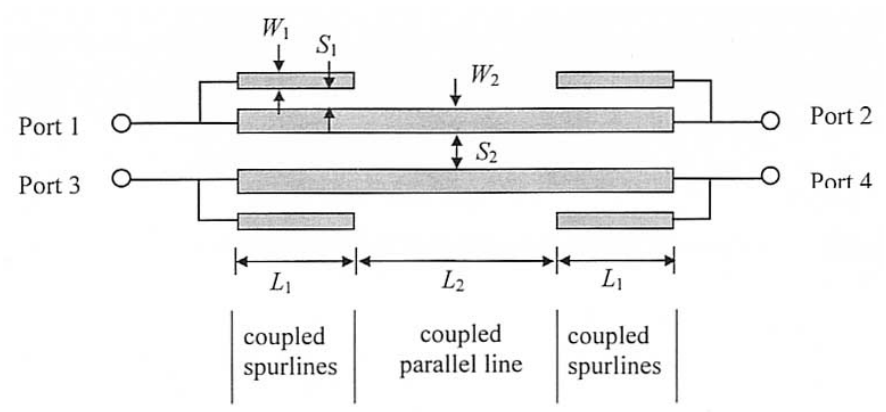

(a)

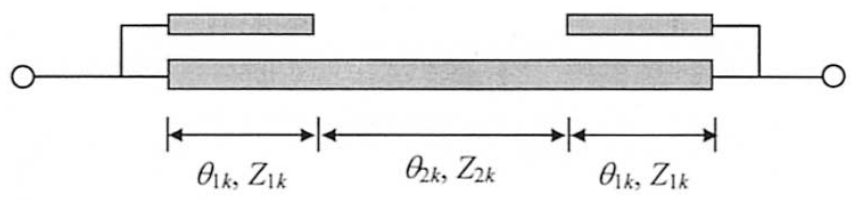

(b)

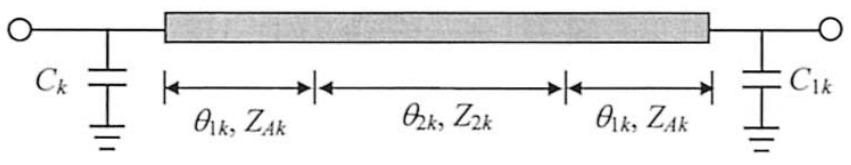

(c)

Fig. 1. Schematic diagram of the proposed spurline coupler, (a) the whole circuit, (b) the reduced two-port circuit $(k=$ ' $e$ ' for the even mode and ' $o$ ' for the odd mode circuit, and (c) the further simplified circuit.

two spurlines inter-connected by a microstrip line, where the spurline is with the coupling coefficient $C_{\text {spur }}$, the electric length $\theta_{1 k}$ and the characteristic impedance $Z_{1 k}$, and the centre microstrip line is with $\theta_{2 k}$ and $Z_{2 k}$. The notation $k=$ ' $e$ ' denotes for the even-mode network and ' $O$ ' for the odd-mode network. For further simplification, the spurline is replaced with a section of microstrip line, $\theta_{1 k}$ and $Z_{A k}$, shunted with a capacitor $C_{k}$ [5]. Then the respective even-mode and odd-mode networks can be represented a cascade of three sections of microstrip lines loaded with the capacitors at both ends, as shown in Fig. 1(c), where

$$
\begin{aligned}
C_{k} & =\sqrt{\frac{\left(1-C_{\mathrm{spur}}\right)^{3}}{1+C_{\mathrm{spur}}}} \frac{1}{\omega Z_{1 k}} \quad \text { and } \\
Z_{A k} & =\frac{Z_{1 k}}{\sqrt{1-C_{\mathrm{spur}}^{2}}} .
\end{aligned}
$$

The loaded capacitor $C_{k}$ increases the effective unit-length capacitance of the cascaded microstrip line so that the phase velocity is reduced. Since the odd-mode impedance $Z_{1 o}$ is 


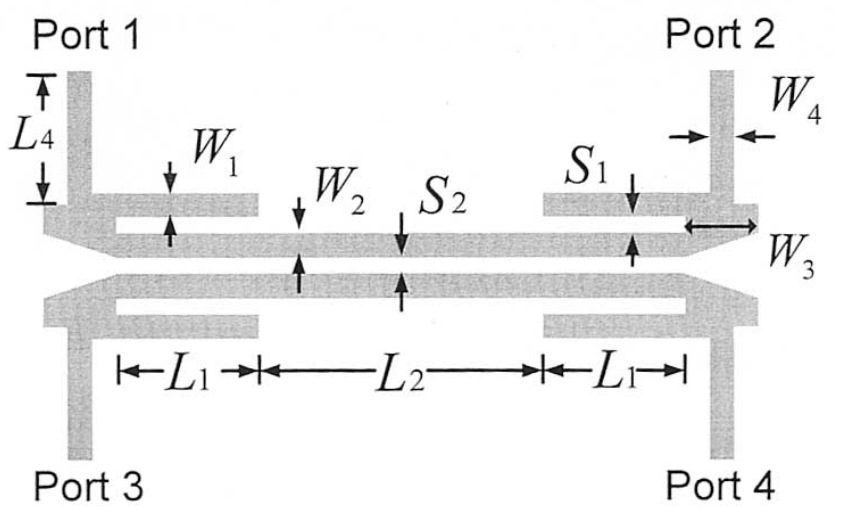

(a)

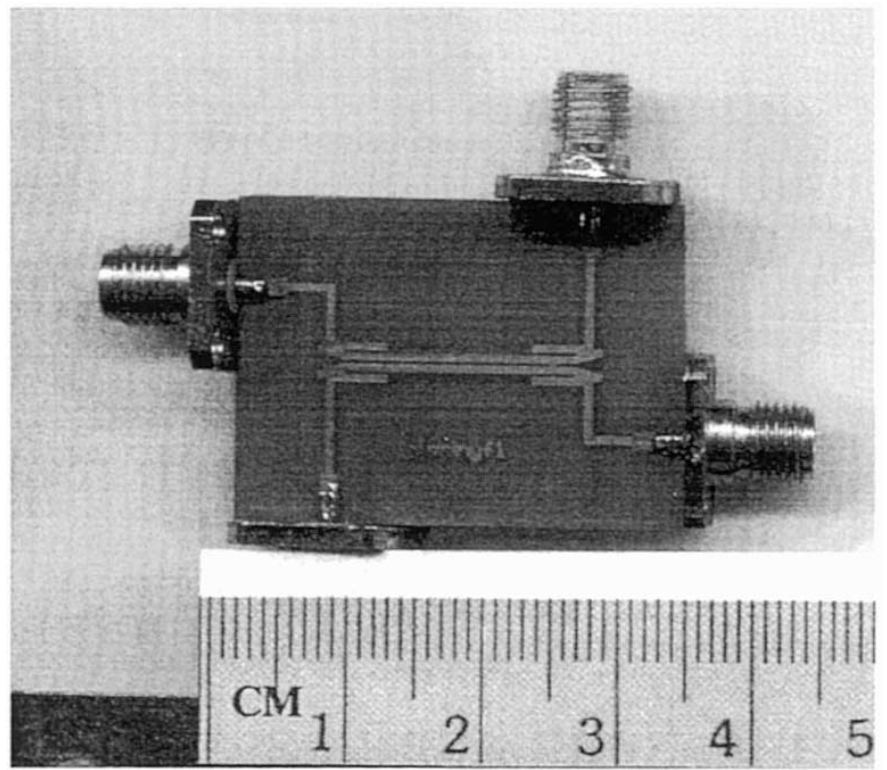

(b)

Fig. 2. (a) Structure in circuit and EM simulations and (b) photograph of the designed spurline coupler.

less than the even-mode impedance $Z_{1 e}$ of the spurline, the odd-mode loaded capacitance $C_{o}$ becomes greater than $C_{e}$. Hence the odd-mode phase velocity is slow down to a greater extent compared with that of the even mode. The equalization of the odd-mode and even-mode velocities can be achieved by properly designing the coupling coefficient $C_{\text {spur }}$ of the spurlines. When the odd-mode and even-mode velocities are equal, an isolation zero is obtained, which in turn results in infinite directivity. Note that the capacitance $C_{k}$ in (1) depends on the frequency, its equalization effect is valid over a limited bandwidth around the center frequency once the coupling coefficient of the spurline is designed.

\section{Simulation And Measurement Results}

To demonstrate the high performance of the proposed spurline coupler, an example of $-10 \mathrm{~dB}$ coupler at $2.4 \mathrm{GHz}$ was designed. In order to obtain accurate design, it is crucial to include the layout discontinuities and nonadjacent coupling. Both the circuit simulator (Microwave Office) and the electromagnetic simulator (IE3D) were performed to optimize the performance. The structure considered in simulations and the

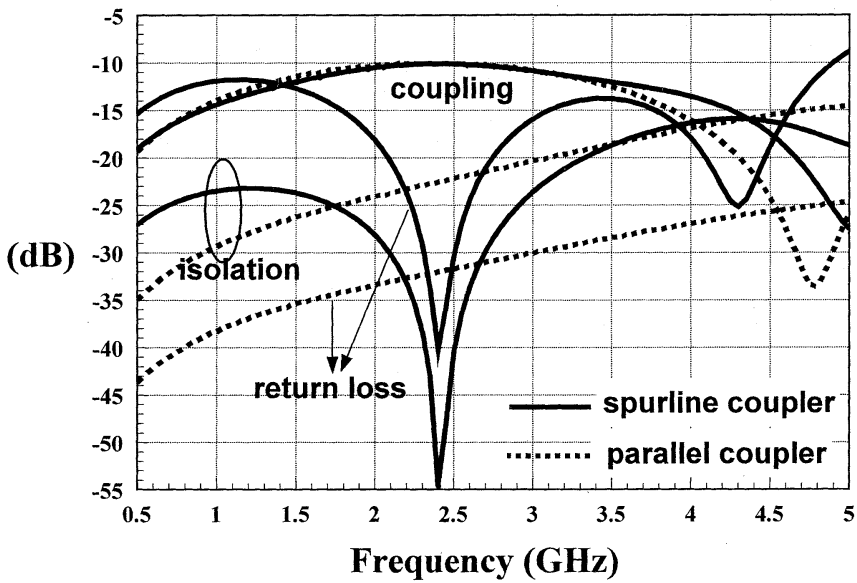

Fig. 3. Simulation performance of the spurline coupler and the conventional parallel-line coupler.

(dB)

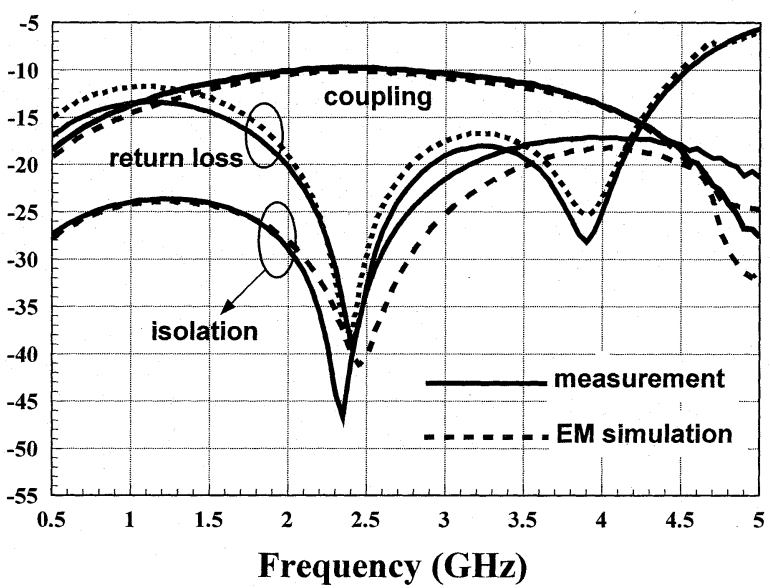

Fig. 4. Measurement and EM simulation performance of the spurline coupler.

circuit photograph are shown in Fig. 2, respectively. During the course of performing the circuit and EM simulations, we find that the isolation zero and input/output matches are sensitive to the connecting junction discontinuities of spurlines with main coupler lines. This results in shifting the frequency of the isolation zero or even diminishing the isolation zero. This effect can be compensated by adding a section of microstrip at each ports of symmetrically coupled spurlines $\left(W_{1}=W_{2}\right.$ and $\left.S_{1}=S_{2}\right)$ or by using asymmetrically coupled spurlines $\left(W_{1} \neq W_{2}\right.$ and $S_{1} \neq S_{2}$ ), where the former method was used in this design example. The physical dimensions of the fabricated spurline coupler are $W_{1}=W_{2}=0.6 \mathrm{~mm}, S_{1}=S_{2}=0.2 \mathrm{~mm}$, $L_{1}=3.3 \mathrm{~mm}, L_{2}=10.2 \mathrm{~mm}, W_{3}=1.8 \mathrm{~mm}, W_{4}=0.6 \mathrm{~mm}$, and $L_{4}=6.2 \mathrm{~mm}$. For comparison, the conventional parallel-line coupler was also made, which has the coupling length $L=17.4 \mathrm{~mm}$, the line-width $W=1.06 \mathrm{~mm}$, and the spacing $S=0.13 \mathrm{~mm}$. Both the circuit sizes are about the same but the proposed spurline coupler has moderate line spacing requirement, which alleviates the fabrication accuracy.

The circuit simulation of the designed spurline coupler from Microwave Office shows that the isolation zero exists at $2.4 \mathrm{GHz}$, as seen from Fig. 3. This significantly improves the isolation around $2.4 \mathrm{GHz}$ compared with the conventional 
parallel coupler. The coupling amplitude of the spurline coupler has flatter response up to $4 \mathrm{GHz}, 0.5-\mathrm{GHz}$ wider than the conventional one. Fig. 4 dictates the measurement and EM simulation results. The isolation zero was measured at $2.35 \mathrm{GHz}$, slightly shifted from the EM simulation. In spite of this frequency shift, the existence of isolation zero enhances the isolation to $47.5 \mathrm{~dB}$, limited by the finite dielectric and conductor losses of the substrate. Good agreement between measurement and simulation is found.

\section{CONCLUSION}

A new high-directivity spurline coupler is proposed, which is composed of two coupled spurlines inter-connected with a parallel-coupled line. The equivalent capacitances of the spurlines slow down the odd and even modes to different extents such that their velocities are equalized once the coupling coefficient of the spurlines is properly designed. The simulation and measurement results all confirm the existence of the existence of isolation zero, which gives a directivity-enhancement around the desired frequency. Flatter coupling response and up to 47.5-dB isolation was achieved compared with the conventional parallel-line coupler. This technique can be applied to multilayer broadside couplers or tight-coupling structures for achievement of high directivity and better output magnitude balance.

\section{REFERENCES}

[1] L. Su, T. Itoh, and J. Rivera, "Design of an overlay directional coupler by a full-wave analysis," IEEE Trans. Microwave Theory Tech., vol. MTT-31, pp. 1017-1022, 1983.

[2] S. Uysal and H. Aghvami, "Synthesis, design and construction of ultrawideband nonuniform directional couplers in inhomogeneous media," IEEE Trans. Microwave Theory Tech., vol. 37, no. 6, pp. 969-976, 1989.

[3] M. Dydyk, "Microstrip directional couplers with ideal performance via single-element compensation," IEEE Trans. Microwave Theory Tech., vol. 47, no. 6, pp. 956-964, 1999.

[4] J.-L. Chen, S.-F. Chang, and C.-T. Wu, "A high-directivity microstrip directional coupler with feedback compensation," in 2002 IEEE MTT-S Int. Microwave Symp. Dig., vol. 1, June 2002, pp. 101-104.

[5] J. A. G. Malherbe, Microwave Transmission Line Filters. Boston, MA: Artech House, 1979. 